COMMUNICATIONS IN

ANALYSIS AND GEOMETRY

Volume 8, Number 4, 795-808, 2000

\title{
Frobenius Manifold Structure on Dolbeault Cohomology and Mirror Symmetry
}

\author{
Huai-Dong CaO AND Jian Zhou
}

We construct a differential Gerstenhaber-Batalin-Vilkovisky algebra from the Dolbeault complex of any closed Kähler manifold, and a formal Frobenius manifold structure on its Dolbeault cohomology.

String theory leads to the mysterious Mirror Conjecture, see Yau [37] for the history. One of the mathematical predictions made by physicists based on this conjecture is the formula due to Candelas-de la Ossa-Green-Parkes [6] on the number of rational curves of any degree on a quintic in $\mathbb{C P}^{4}$. Recently, it has been proved by Lian-Liu-Yau [20], completing the program of Kontsevich, Manin and Givental.

The theory of quantum cohomology, also suggested by physicists, has lead to a better mathematical formulation of the Mirror Conjecture. As explained in Witten [38], there are two topological conformal field theories on a Calabi-Yau manifold $X$ : the A theory is independent of the complex structure of $X$, but depends on the Käher form on $X$, while the B theory is independent of the Kähler form of $X$, but depends on the complex structure of $X$. Vafa [33] explained how two quantum rings $\mathcal{R}_{x}$ and $\mathcal{R}_{x}{ }^{\prime}$ arise from these theories, and the notion of mirror symmetry could be translated into the equivalence of A theory on a Calabi-Yau manifold $X$ with B theory on another Calabi-Yau manifold $\hat{X}$, called the mirror of $X$, in the sense that the quantum ring $\mathcal{R}_{x}$ can be identified with $\mathcal{R}_{x}{ }^{\prime}$.

Earlier interpretation of mirror symmetry exploits variations of Hodge structures, see e.g. Morrison [25] or Bertin-Peters [4]. There are two natural Frobenius algebras on any Calabi-Yau $n$-fold,

$$
A(X)=\oplus_{k=0}^{n} H^{k}\left(X, \Omega^{k}\right), \quad B(X)=\oplus_{k=0}^{n} H^{k}\left(X, \Omega^{-k}\right),
$$

where $\Omega^{-k}$ is the sheaf of holomorphic sections to $\Lambda^{k} T X$. By Hodge theory, $B(X)$ can be identified with $H^{n}(X, \mathbb{C})$. By the Bogomolov-Tian-Todorov

\footnotetext{
${ }^{1}$ Both authors were supported in part by NSF
} 
theorem, there is a deformation of the complex structures on $X$ parameterized by an open set in $H^{1}\left(X, \Omega^{-1}\right)$. Therefore, one gets a family of Frobenius algebra structures on $H^{n}(X, \mathbb{C})$. Every Frobenius algebra structure can be characterized by a cubic polynomial $\Phi$ (in Physics literature, it is called the Yukawa coupling), so we get a family $\Phi_{B}(X)$ of cubic polynomials on $H^{n}(X, \mathbb{C})$ parameterized by an open set in $H^{1}\left(X, \Omega^{-1}\right)$. An additional structure provided by algebraic geometry is the Gauss-Manin connection on this family. It is a flat connection with some extra properties. On the other hand, counting of rational curves provides a family $\Psi_{A}(X)$ of cubic polynomials on $A(X)$ parameterized by an open set in $H^{1}\left(X, \Omega^{1}\right)$. One version of Mirror Conjecture is the conjectural existence, for a Calabi-Yau 3-fold $X$, of another Calabi-Yau 3-fold $\hat{X}$, such that one can identify $B(X)$ with $A(\hat{X})$, $\Phi_{B}(X)=\Psi_{A}(\hat{X})$, and vice versa.

However, the $A$ theory and the $B$ theory should provide deformations of

$$
\widetilde{A}(X)=\oplus_{p, q \geq 0} H^{p}\left(X, \Omega^{q}\right), \quad \text { and } \quad \widetilde{B}(X)=\oplus_{p, q \geq 0} H^{p}\left(X, \Omega^{-q}\right),
$$

which are parameterized by open neighborhood of 0 in $\widetilde{A}(X)$ and $\widetilde{B}(X)$ respectively. According to Dijkgraaf-E. Verlinde-H. Verlinde [10] and Witten [35], the associativity condition can be encoded in a system of nonlinear equations called WDVV (Witten-Dijkgraaf-Verlinde-Verlinde) equations. Motivated by this, Dubrovin [11, 12] introduced and extensively studied the notion of Frobenius manifolds. On the $A$ side, under the influence of Witten [35], Ruan-Tian [28] gave a mathematical formulation of quantum cohomology using Gromov-Witten invariants (see also Liu [21] and McDuffSalamon [22]). They also proved WDVV equations for the quantum cohomology. Witten [38] suggested two kinds of extended moduli spaces, one containing the deformation space of the complex structure, the other containing the complexified Kähler cone. The former provides a natural setting for the $B$ side of the story. Extended moduli space of complex structures on a Calabi-Yau manifold was studied by physicists in the Kodaira-Spencer theory of gravity (Bershadsky-Ceccoti-Ooguri-Vafa [3]). As a generalization of the Bogomolov-Tian-Todorov theorem, they showed that the extended moduli space locally is an open subset of the supermanifold $H^{-*, *}(M)$. For related work, see Ran [27] and Gerstenhaber-Schack [13]. Recently, Barannikov-Kontsevich [2] constructed a structure of formal Frobenius manifold on $H^{-*, *}(M)$ based on the above works. This construction and its application to mirror symmetry was known to the authors of [3]. It was singled out among many impressive results in [3], and given a mathematical treatment. The remark in [2] that this construction can be carried out 
for any differential Gerstenhaber-Batalin-Vilkovisky (dGBV) algebra with suitable conditions was treated with more details in Manin [24].

The purpose of this paper is to construct a formal Frobenius manifold structure on Dolbeault cohomology $H^{*, *}(M)$ of a closed Kähler manifold by dGBV algebra approach. Comparison with that of Barannikov-Kontsevich [2] suggests that these two kinds of formal Frobenius manifolds might be isomorphic with each other for a pair of mirror manifolds. Now we have two formal Frobenius manifolds on the $A$-side: one from counting rational curves by Gromov-Witten invariants, the other from our construction. From consideration of spectrum of the Frobenius manifolds, it is known if the formal Frobenius manifolds constructed from a dGBV algebra can be identified with the formal Frobenius manifold structure on the de Rham cohomology of a symplectic manifold $M$, then $M$ must have torsion $c_{1}$. Nevertheless, it is reasonable to conjecture that for a Calabi-Yau manifold $M$, the Frobenius manifold structure we construct on $H^{*, *}(M)$ can be identified with that from quantum cohomology. If this is true, it should have some applications in enumerative geometry of Calabi-Yau manifolds.

Acknowledgements. The authors would like to thank Jim Stasheff, Gang Tian and Shing-Tung Yau for their interest. The work in this paper is carried out while the second author is visiting Texas A\&M University. He likes to express his appreciation for the hospitality and financial support of the Mathematics Department and the Geometry-Analysis-Topology group. He also likes to thank Blaine Lawson for bringing his attention to the reference [17], which made him come across [2] and [24] on the internet.

\section{Frobenius algebras and Frobenius manifolds.}

In this section, we review the definition of (formal) Frobenius manifold. We will only be concerned with a special case which comes from the consideration of deformations of Frobenius algebras. For the general case, see Dubrovin [12] and Manin [23].

Let $H$ be a finite dimensional commutative associative algebra with 1 over $\mathbf{k}=\mathbb{R}$ or $\mathbb{C}$ with multiplication denoted by $\cdot$. Take a basis $\left\{e_{a}\right\}$ of $H$ such that $e_{0}=1$. Then there are constants $\phi_{a b}^{c} \in \mathbf{k}$ such that

$$
e_{a} \cdot e_{b}=\phi_{a b}^{c} e_{c}
$$

An inner product on $H$ is a nondegenerate quadratic form

$$
(\cdot, \cdot): H \times H \rightarrow \mathbf{k}
$$


It is called invariant if

$$
(a \cdot b, c)=(a, b \cdot c),
$$

for any $a, b, c \in H$. A commutative associative algebra with 1 together with an invariant inner product is called a Frobenius algebra. A simple observation is that the structure of a Frobenius algebra $H$ can be encoded in a cubic polynomial $\phi: H \rightarrow \mathbf{k}$ as follows. Let $\eta_{a b}=\left(e_{a}, e_{b}\right)$ and $\left(\eta^{a b}\right)$ be the inverse matrix of $\left(\eta_{a b}\right)$. Set $\phi_{a b c}=\phi_{a b}^{p} \eta_{p c}$. Then

$$
\phi_{a b c}=\left(e_{a} \cdot e_{b}, e_{c}\right) .
$$

Hence (1) implies that $\phi$ is symmetric in the three indices. One can recover the inner product and multiplication by

$$
\eta_{a b}=\phi_{0 a b}, \quad \phi_{a b}^{c}=\phi_{a b p} \eta^{p c} .
$$

The associativity of the multiplication is equivalent to the following system of equations

$$
\phi_{a b p} \eta^{p q} \phi_{q c d}=\phi_{b c p} \eta^{p q} \phi_{a q d}
$$

Consider a smooth (analytic) family of associativity multiplications $\left\{\cdot_{\alpha}\right.$ : $\alpha \in H\}$ such that ${ }_{0}=\cdot$. Then we get a family of structure constants $\phi_{a b c}(\alpha)$ such that $\phi_{a b c}(0)=\phi_{a b c}$. Denote by $\left\{x^{a}\right\}$ the linear coordinates in the basis $\left\{e_{a}\right\}$. If for any $\alpha \in H$,

$$
\phi_{a b c, d}(\alpha)=\frac{\partial}{\partial x^{d}} \phi_{a b c}(\alpha)
$$

is symmetric in all four indices, then there is a function $\Phi: H \rightarrow \mathbf{k}$, such that

$$
\phi_{a b c}(\alpha)=\frac{\partial^{3} \Phi}{\partial x^{a} \partial x^{b} \partial x^{c}}(\alpha) .
$$

The function $\Phi$ is called the potential function of the family $(H,\{\cdot \alpha\})$ which is then called a potential family. It is clear that

$$
\left(\frac{\partial^{3} \Phi}{\partial x^{0} \partial x^{a} \partial x^{b}}(\alpha)\right)=\left(\eta_{a b}\right)
$$

is a constant symmetric nondegenerate matrix if 1 is an identity for all $\alpha$. Furthermore, from (2), we know that the associativity of the multiplications $\cdot_{\alpha}$ implies that $\Phi$ satisfies the WDVV equations:

$$
\frac{\partial^{3} \Phi}{\partial x^{a} \partial x^{b} \partial x^{p}} \eta^{p q} \frac{\partial^{3} \Phi}{\partial x^{q} \partial x^{c} \partial x^{d}}=\frac{\partial^{3} \Phi}{\partial x^{b} \partial x^{c} \partial x^{p}} \eta^{p q} \frac{\partial^{3} \Phi}{\partial x^{a} \partial x^{q} \partial x^{d}} .
$$


Conversely, given a Frobenius algebra $(H, \cdot(\cdot, \cdot))$, a function $\Phi$ satisfying (3), (4) and such that

$$
\phi_{a b c}=\frac{\partial^{3} \Phi}{\partial x^{a} \partial x^{b} \partial x^{c}}(0),
$$

we have a potential family of Frobenius algebra structures on $H$ with the fixed inner product. This gives an example of a Frobenius manifold structure on $H$. If $\Phi$ is just a formal power series with the above properties, then it yield a formal Frobenius manifold structure.

Remark 1.1. One can also consider $\mathbb{Z}_{2}$-graded version of the above discussion. For details, cf. Manin [23, 24].

\section{A construction of formal Frobenius (super)manifolds.}

In this section, we review a construction of formal Frobenius supermanifolds. For details, the reader should consult the papers by Tian [31], Todorov [32], Bershadsky-Ceccoti-Ooguri-Vafa [3], Barannikov-Kontsevich [2] and Manin [24]. Here, we follow the formulation by Manin [24].

Let $(\mathcal{A}, \wedge)$ be a supercommutative associative algebra with identity over a field $\mathbf{k}$, i.e., $(\mathcal{A}, \wedge)$ is an algebra with identity over $\mathbf{k}$, furthermore, $\mathcal{A}$ has $\mathbb{Z}_{2}$-grading, such that for any homogeneous elements $a, b \in \mathcal{A}$ with degrees $|a|,|b|$ respectively, we have

$$
a \wedge b=(-1)^{|a| \cdot|b|} b \wedge a .
$$

Assume that there is a $\mathbf{k}$-linear map $\delta$ of odd degree on $\mathcal{A}$, such that $\delta^{2}=0$, and $\delta$ is a derivation: $\delta(a \wedge b)=(\delta a) \wedge b+(-1)^{|a|} a \wedge(\delta b)$. Assume that the cohomology $H=H(\mathcal{A}, \delta)$ is finite dimensional. Then there is an induced multiplication $\wedge$ on $H$. We will be interested in the deformation of this multiplication obtained by deforming the operator $\delta$.

For this idea to work, one needs the notion of a differential GerstenhaberBatalin-Vilkovisky (dGBV) algebra (see e.g. [24], §5). More precisely, one needs another $\mathbf{k}$-linear map $\Delta$ of odd degree on $\mathcal{A}$, such that

- $\delta \Delta+\Delta \delta=0, \Delta^{2}=0$.

- If $[a \bullet b]=(-1)^{|a|}\left(\Delta(a \wedge b)-\Delta a \wedge b-(-1)^{|a|} a \wedge \Delta b\right)$, then $[a \bullet \cdot]: \mathcal{A} \rightarrow \mathcal{A}$ is a derivation of degree $|a|+1$, i.e.,

$$
[a \bullet(b \wedge c)]=[a \bullet b] \wedge c+(-1)^{(|a|+1)|b|} b \wedge[a \bullet c] .
$$


The quintuple $(\mathcal{A}, \wedge, \delta, \Delta,[\cdot \bullet \cdot])$ with the above properties is called a dGBV algebra. As in Manin [24], §5, we have

$$
\begin{aligned}
{[a \bullet b] } & =-(-1)^{(|a|+1)(|b|+1)}[b \bullet a], \\
{[a \bullet[b \bullet c]] } & =[[a \bullet b] \bullet c]+(-1)^{(|a|+1)(|b|+1)}[b \bullet[a \bullet c]], \\
\Delta[a \bullet b] & =[\Delta a \bullet b]+(-1)^{|a|+1}[a \bullet \Delta b], \\
\delta[a \bullet b] & =[\delta a \bullet b]+(-1)^{|a|+1}[a \bullet \delta b] .
\end{aligned}
$$

See also Koszul [18].

An integral on a dGBV algebra $\mathcal{A}$ is an even linear functional $\int: \mathcal{A} \rightarrow \mathbf{k}$, such that

$$
\begin{aligned}
& \int(\delta a) \wedge b=(-1)^{|a|+1} \int a \wedge(\delta b), \\
& \int(\Delta a) \wedge b=(-1)^{|a|} \int a \wedge(\Delta b),
\end{aligned}
$$

for any homogeneous $a, b \in \mathcal{A}$. It follows from (5) that $\int$ induces a welldefined $\mathbf{k}$-bilinear functional

$$
\begin{aligned}
& (\cdot, \cdot): H(\mathcal{A}, \delta) \otimes H(\mathcal{A}, \delta) \rightarrow \mathbf{k}, \\
& ([\alpha],[\beta])=\int \alpha \wedge \beta,
\end{aligned}
$$

where $[\alpha]$ and $[\beta]$ are cohomology classes represented by $\delta$-closed $\alpha$ and $\beta$ respectively. If $(\cdot, \cdot)$ is nondegenerate, we say that the integral is nice. It is obvious that

$$
([\alpha] \wedge[\beta],[\gamma])=([\alpha],[\beta] \wedge[\gamma]) .
$$

By definition, $(H(\mathcal{A}, d), \wedge,(\cdot, \cdot))$ is then a Frobenius algebra, when $\mathcal{A}$ has a nice integral.

To find a deformation of the ring structure on $H$, consider $\delta_{a}=\delta+[a \bullet \cdot]$ : $\mathcal{A} \rightarrow \mathcal{A}$ for even $a \in \mathcal{A}$. When $a$ satisfies

$$
\begin{aligned}
& \delta a+\frac{1}{2}[a \bullet a]=0, \\
& \Delta a=0,
\end{aligned}
$$

then $\left(\mathcal{A}, \wedge, \delta_{a}, \Delta,[\cdot \bullet \cdot]\right)$ is also a dGBV algebra. Such deformations appeared also in e.g. Akman [1], §5.3. If $\int$ is an integral for $(\mathcal{A}, \wedge, \delta, \Delta,[\bullet \bullet \cdot)$, so is it for $\left(\mathcal{A}, \wedge, \delta_{a}, \Delta,[\cdot \bullet \cdot]\right)$. If there is a natural way to identify $H\left(\mathcal{A}, \delta_{a}\right)$ with $H=H(\mathcal{A}, \delta)$ which preserves $(\cdot, \cdot)$, then we get another Frobenius 
algebra structure on $H$. The construction of Frobenius manifold structure is based on the existence of a solution $\Gamma=\sum \Gamma_{n}$ to (7), such that $\Gamma_{0}=0$, $\Gamma_{1}=\sum x^{j} e_{j},\left\{e_{j} \in \operatorname{Ker} \delta \cap \operatorname{Ker} \Delta\right\}$ induce a basis of $H$. For $n>1, \Gamma_{n} \in \operatorname{Im} \Delta$ is a homogeneous super polynomial of degree $n$ in $x^{j}$ 's, such that the total degree of $\Gamma_{n}$ is even. Furthermore, $x^{0}$ only appears in $\Gamma_{1}$. Such a solution is called a normalized universal solution. Its existence can be established inductively. This is how Tian [30] and Todorov [32] proved that the deformation of complex structures on a Calabi-Yau manifold is unobstructed. This was generalized by Bershadsky et al [3] to the case of extended moduli space of complex structures of a Calabi-Yau manifold. An interesting observation in [3] is that the action functional of the Kodaira-Spencer theory of gravity provides the potential function for a formal structure on $H^{-* . *}(x)$. As mentioned earlier, Barannikiv and Kontesevich [2] gave a mathematical treatment of this observation. They also remarked that this construction can be carried out for dGBV algebra with suitable condition. See Manin [24] for a detailed account. The result can be stated as follows:

Theorem 2.1. Let $(\mathcal{A}, \wedge, \delta, \Delta,[\cdot \bullet \cdot)$ be a $d G B V$ algebra which satisfies the following conditions:

1. $H=H(\mathcal{A}, \delta)$ is finite dimensional.

2. There is a nice integral on $\mathcal{A}$.

3. The inclusions $i:(\operatorname{Ker} \Delta, \delta) \hookrightarrow(\mathcal{A}, \delta)$ and $j:(\operatorname{Ker} \delta, \Delta) \hookrightarrow(\mathcal{A}, \Delta)$ induce isomorphisms on cohomology.

Then there is a structure of formal Frobenius manifold on the formal spectrum of $\mathbf{k}\left[\left[H^{\prime}\right]\right]$, the algebra of formal power series generated by $H^{\prime}$, where $H^{\prime}$ is the dual $\mathbf{k}$-vector space of $H$.

\section{Frobenius manifold structures on Dolbeault cohomology.}

In this section, we show that there is a natural dGBV algebra structure on the Dolbeault complex of a Kähler manifold. Furthermore, it is shown that all the conditions in Theorem 2.1 are satisfied.

Let $(X, g, J)$ be a closed Kähler manifold with Kähler form $\omega$. Consider the quadruple $\left(\Omega^{*, *}(X), \wedge, \delta=\bar{\partial}, \Delta=\partial^{*}\right)$. It is well-known that $\bar{\partial}^{2}=0$, $\left(\partial^{*}\right)^{2}=0$, and $\bar{\partial} \partial^{*}+\partial^{*} \bar{\partial}=0$. Furthermore, $\bar{\partial}$ is a derivation. Set

$$
[a \bullet b]_{\partial^{*}}=(-1)^{|a|}\left(\partial^{*}(a \wedge b)-\partial^{*} a \wedge b-(-1)^{|a|} a \wedge \partial^{*} b\right) .
$$


A formula of this type in complex geometry was discovered by Tian [30] to prove the important result that deformations of Calabi-Yau manifolds are unobstructed (cf. Todorov [32] and Bershadsky et al [3]). In Tian's situation, he used the holomorphic volume form on a Calabi-Yau manifold to get an operator on $\Omega^{-*, *}(M)$ from $\partial$ on $\Omega^{*, *}(M)$ and showed that the corresponding bracket can be identified with the Schouten-Nijenhuis bracket. Such a method of defining bracket also appeared in Koszul's work on Poisson geometry [18]. Conjugation of the exterior differential $d$ by the isomorphism induced by a volume form was used in Witten [38] and Schwarz [29] to construct BV algebras.

Since both $\omega$ and $J$ are parallel with respect to the Levi-Civita connection, it follows that near each $x \in X$, one can find a local frame $\left\{e_{1}, \cdots, e_{n}\right\}$ of $T^{1,0} X$ with dual frame $\left\{e^{1}, \cdots, e^{n}\right\}$, such that $\omega=e^{1} \wedge \bar{e}^{1}+\cdots+e^{n} \wedge \bar{e}^{n}$. Furthermore, $\nabla_{e_{j}} e_{k}=\nabla_{\bar{e}_{j}} e_{k}=\nabla_{e_{j}} \bar{e}_{k}=\nabla_{\bar{e}_{j}} \bar{e}_{k}=0$ at $x$. Then at $x$, for any $\alpha \in \Omega^{* * *}(X)$, we have (Griffiths-Harris [15], p. 113):

$$
\begin{aligned}
\partial \alpha=e^{j} \wedge \nabla_{e_{j}} \alpha, & \bar{\partial} \alpha=\bar{e}^{j} \wedge \nabla_{\bar{e}_{j}} \alpha, \\
\partial^{*} \alpha=-e_{j} \vdash \nabla_{\bar{e}_{j}} \alpha, & \bar{\partial}^{*} \alpha=-\bar{e}_{j} \vdash \nabla_{e_{j}} \alpha,
\end{aligned}
$$

Lemma 3.1. $[a \bullet(b \wedge c)]_{\partial^{*}}=[a \bullet b]_{\partial^{*}} \wedge c+(-1)^{(|a|+1)|b|} b \wedge[a \bullet c]_{\partial^{*}}$.

Proof. We first express $[a \bullet b]_{\partial^{*}}$ in terms of the Levi-Civita connection:

$$
\begin{aligned}
{[a \bullet b]_{\partial^{*}}=} & (-1)^{|a|}\left(\partial^{*}(a \wedge b)-\left(\partial^{*} a\right) \wedge b-(-1)^{|a|} a \wedge \partial^{*} b\right) \\
= & (-1)^{|a|}\left(\partial^{*}(a \wedge b)-\left(\partial^{*} a\right) \wedge b-(-1)^{|a|} a \wedge \partial^{*} b\right) \\
= & (-1)^{|a|}\left(-\left(e_{j} \vdash \nabla_{\bar{e}_{j}}(a \wedge b)\right)+\left(e_{j} \vdash \nabla_{\bar{e}_{j}} a\right) \wedge b\right. \\
& \left.+(-1)^{|a|} a \wedge\left(e_{j} \vdash \nabla_{\bar{e}_{j}} b\right)\right) \\
= & -(-1)^{|a|} e_{j} \vdash\left(\nabla_{\bar{e}_{j}} a \wedge b+a \wedge \nabla_{\bar{e}_{j}} b\right)+(-1)^{|a|}\left(e_{j} \vdash \nabla_{\bar{e}_{j}} a\right) \wedge b \\
& +a \wedge\left(e_{j} \vdash \nabla_{\bar{e}_{j}} b\right) \\
= & -\nabla_{\bar{e}_{j}} a \wedge\left(e_{j} \vdash b\right)-(-1)^{|a|}\left(e_{j} \vdash a\right) \wedge \nabla_{\bar{e}_{j}} b .
\end{aligned}
$$

The left hand side is then given by

$$
[a \bullet(b \wedge c)]_{\partial^{*}}=-\nabla_{\bar{e}_{j}} a \wedge e_{j} \vdash(b \wedge c)-(-1)^{|a|}\left(e_{j} \vdash a\right) \wedge \nabla_{\bar{e}_{j}}(b \wedge c) .
$$


The right hand side is computed in a similar fashion:

$$
\begin{aligned}
{[a \bullet b]_{\partial^{*}} \wedge } & c+(-1)^{(|a|+1)|b|} b \wedge[a \bullet c]_{\partial^{*}} \\
= & \left(-\nabla_{\bar{e}_{j}} a \wedge\left(e_{j} \vdash b\right)-(-1)^{|a|}\left(e_{j} \vdash a\right) \wedge \nabla_{\bar{e}_{j}} b\right) \wedge c \\
& +(-1)^{(|a|+1)|b|} b \wedge\left(-\nabla_{\bar{e}_{j}} a \wedge\left(e_{j} \vdash c\right)-(-1)^{|a|}\left(e_{j} \vdash a\right) \wedge \nabla_{\bar{e}_{j}} c\right) \\
= & -\nabla_{\bar{e}_{j}} a \wedge\left(e_{j} \vdash b\right) \wedge c-(-1)^{|a|}\left(e_{j} \vdash a\right) \wedge \nabla_{\bar{e}_{j}} b \wedge c \\
& -(-1)^{|b|} \nabla_{\bar{e}_{j}} a \wedge b \wedge\left(e_{j} \vdash c\right)-(-1)^{|a|}\left(e_{j} \vdash a\right) \wedge b \wedge \nabla_{\bar{e}_{j}} c \\
= & -\nabla_{\bar{e}_{j}} a \wedge e_{j} \vdash(b \wedge c)-(-1)^{|a|}\left(e_{j} \vdash a\right) \wedge \nabla_{\bar{e}_{j}}(b \wedge c) .
\end{aligned}
$$

This completes the proof.

Corollary 3.1. For any Kähler manifold $(X, g),\left(\Omega^{*, *}(X), \wedge, \delta=\bar{\partial}, \Delta=\right.$ $\left.\partial^{*}\right)$ is a $d G B V$ algebra.

Remark 3.1. It is actually more natural to take

$$
\Delta=-\sqrt{-1} \partial^{*} .
$$

Let $\int_{X}: \Omega^{*, *}(X) \rightarrow \mathbb{C}$ be the ordinary integration of differential forms. It is easy to show the following

Lemma 3.2. The following identity holds on closed Kähler manifolds:

$$
\begin{aligned}
& \int_{X} \bar{\partial} a \wedge b=(-1)^{|a|+1} \int_{X} a \wedge \bar{\partial} b \\
& \int_{X} \partial^{*} a \wedge b=(-1)^{|a|} \int_{X} a \wedge \partial^{*} b .
\end{aligned}
$$

Theorem 3.1. For any closed Kähler manifold $X$, if $K$ is the algebra of formal power series generated by the dual space of the Dolbeault cohomology $H_{\bar{\partial}}^{*}(X)$, then there is a structure of formal Frobenius manifold on the formal spectrum of $K$ obtained from the $d G B V$ algebra $\left(\Omega^{* * *}(X), \wedge, \delta=\bar{\partial}, \Delta=\partial^{*}\right)$.

Proof. By the method of Deligne-Griffiths-Morgan-Sullivan [9], the two natural inclusions $i:\left(\operatorname{Ker} \partial^{*}, \bar{\partial}\right) \rightarrow\left(\Omega^{* * *}(X), \bar{\partial}\right)$ and $j:\left(\operatorname{Ker} \bar{\partial}, \partial^{*}\right) \rightarrow$ $\left(\Omega^{*, *}(X), \partial^{*}\right)$ induce isomorphisms on cohomology. Then the theorem follows from Lemma 3.2 and Theorem 2.1 .

Remark 3.2. The same construction carries through if we take $\delta=\partial$, $\Delta=\bar{\partial}^{*}$. 


\section{Comparison with Barannikov-Kontsevich's Frobenius manifold.}

In this section, we compare our construction with that of BarannikovKontsevich [2], and make some speculations based on their similarity.

Assume that $X$ is a closed Calabi-Yau $n$-manifold; fix a nowhere vanishing holomorphic $n$-form $\Omega \in \Gamma\left(X, \Lambda^{n} T^{*} X\right)$. Barannikov-Kontsevich [2] considered the dGBV algebra with

$$
\mathcal{B}=\oplus \mathcal{B}_{k}, \quad \mathcal{B}_{k}=\oplus_{q+p=k} \Gamma\left(X, \Lambda^{p} T X \otimes \Lambda^{q} \bar{T}^{*} X\right) .
$$

Here $\mathcal{B}$ is $\mathbb{Z}$-graded. It also has an induced $\mathbb{Z}_{2}$-grading. This grading is different from that in [2] (shifted by 1); we adopt this grading to be compatible with the notations of Manin [24]. The multiplication $\wedge$ on $\mathcal{B}$ is given by the ordinary wedge products on $\Lambda^{*} \bar{T}^{*} X$ and $\Lambda^{*} T X$. The derivation is $\delta=\bar{\partial}$. Notice that for any two integers $0 \leq p, q \leq n, \Omega$ defines an isomorphism

$$
\Lambda^{p} T X \otimes \Lambda^{q} \bar{T}^{*} X \rightarrow \Lambda^{n-p} T^{*} X \otimes \Lambda^{q} \bar{T}^{*} X, \quad \gamma \mapsto \gamma \vdash \Omega,
$$

defined by the contraction of the $p$-vector with $\Omega$ to get a form of type $(n-p, 0)$. Then $\Delta$ is defined by

$$
(\Delta \gamma) \vdash \Omega=\partial(\gamma \vdash \Omega)
$$

Tian's formula shows that the bracket, defined by

$$
[\alpha \bullet \beta]=(-1)^{|\alpha|}\left(\Delta(\alpha \wedge \beta)-\Delta \alpha \wedge \beta-(-1)^{|\alpha|} \alpha \wedge \Delta \beta\right),
$$

is given by the wedge product on type $(0, *)$-forms and the SchoutenNijenhuis bracket on type $(*, 0)$ polyvector fields. Hence $(\mathcal{B}, \wedge, \delta, \Delta)$ as above is a dGBV algebra. Furthermore, the linear functional

$$
\int: \mathcal{B} \rightarrow \mathbb{C}, \quad \quad \int \gamma=\int_{X}(\gamma \vdash \Omega) \wedge \Omega
$$

is a nice integral on $X$.

Since the complex $(\mathcal{B}, \delta)$ contains the deformation complex

$$
\Omega^{0,0}(T X) \stackrel{\bar{\partial}}{\rightarrow} \Omega^{0,1}(T X) \stackrel{\bar{\partial}}{\rightarrow} \Omega^{0,2}(T X),
$$

it is called the extended deformation complex. Barannikov-Kontsevich [2] (Lemma 2.1) showed that the deformation functor associated with the graded differential Lie algebra $(\mathcal{B}[-1], \delta,[\bullet \bullet \cdot])$ is represented by the formal 
spectrum of formal power series generated by $H^{\prime}$, the dual of $H=H(\mathcal{B}, \delta)$. (This is the extended moduli space of complex structures in Witten [38].) Their method is that used in [31], [32] and [3].

There are remarkable similarities between the dGBV algebras used by us and in [2]. One can also express $\Delta$ on $\mathcal{B}$ in terms of Levi-Civita connection:

$$
\Delta=e^{j} \vdash \nabla_{j} .
$$

We have the following interesting comparison of the two dGBV algebras:

$$
\begin{array}{llrr}
(\mathrm{BK}) & \mathcal{B}=\oplus_{p, q} \Gamma\left(X, \Lambda^{p} T X \otimes \Lambda^{q} \bar{T}^{*} X\right), & \delta=\bar{\partial}, & \Delta=e^{j} \vdash \nabla_{j}, \\
(\mathrm{CZ}) & \mathcal{A}=\oplus_{p, q} \Gamma\left(X, \Lambda^{p} T^{*} X \otimes \Lambda^{q} \bar{T}^{*} X\right), & \delta=\bar{\partial}, & \Delta=\partial^{*}=-e_{j} \vdash \nabla_{j},
\end{array}
$$

(The multiplications on both algebras are given by the exterior products!) It is reasonable to conjecture that the two corresponding Frobenius manifolds are isomorphic to each other for a pair of mirror manifolds.

\section{References.}

[1] F. Akman, On some generalizations of Batalin-Vilkovisky algebras, J. Pure Appl. Algebra, 120 (1997), no. 2, 105-141.

[2] S. Barannikov, M. Kontsevich, Frobenius Manifolds and Formality of Lie Algebras of Polyvector Fields, Internat. Math. Res. Notices 1998, no. 4, 201-215, alg-geom/9710032.

[3] M. Bershadsky, S. Ceccoti, H. Ooguri, C. Vafa, Kodaira-Spencer theory of gravity and exact results for quantum string amplitudes Comm. Math. Phys. 165 (1994), no. 2, 311-427.

[4] J. Bertin, C. Peters, Variations de structures de Hodge, variétés de CalabiYau et symétrie miroir, in Introduction à la théorie de Hodge, 169-256, Panor. Synthèses, 3, Soc. Math. France, Paris, 1996.

[5] J.-L. Brylinski, A differential complex for Poisson manifolds, J. Differential Geom. 28 (1988), no. 1, 93-114.

[6] P. Candelas, X. C. de la Ossa, p. S. Green, L. Parkes, A pair of Calabi-Yau manifolds as an exactly soluble superconformal theory, Nuclear Phys. B, 359 (1991), no. 1, 21-74.

[7] H.-D. Cao, J. Zhou, On Quantum de Rham Cohomology Theory, preprint, April, 1998.

[8] H.-D. Cao, J. Zhou, Identification of Two Frobenius Manifolds, Math. Res. Lett. 6 (1999), no. 1, 17-29. 
[9] P. Deligne, P. Griffiths, J. Morgan, D. Sullivan, Real homotopy theory of Kähler manifolds, Invent. Math. 29 (1975), no. 3, 245-274.

[10] R. Dijkgraaf, H. Verlinde, E. Verlinde, Notes on topological string theory and $2 D$ quantum gravity, in String theory and quantum gravity (Trieste, 1990), 91-156, World Sci. Publishing, River Edge, NJ, 1991.

[11] B. Dubrovin, Integrable systems in topological field theory, Nuclear Phys. B, 379 (1992), no. 3, 627-689.

[12] B. Dubrovin, Geometry of $2 D$ topological field theories, in Integrable systems and quantum groups (Montecatini Terme, 1993), 120-348, Lecture Notes in Math., 1620, Springer, Berlin, 1996.

[13] M. Gerstenhaber, S.D. Schack, Algebraic cohomology and deformation theory, in Deformation theory of algebras and structures and applications (Il Ciocco, 1986), 11-264, NATO Adv. Sci. Inst. Ser. C: Math. Phys. Sci., 247, Kluwer Acad. Publ., Dordrecht, 1988.

[14] W.M. Goldman, J.J. Millson, The deformation theory of representations of fundamental groups of compact Khler manifolds, Inst. Hautes tudes Sci. Publ. Math. 67 (1988), 43-96.

[15] P. Griffiths, J. Harris, Principles of algebraic geometry, Pure and Applied Mathematics, Wiley-Interscience [John Wiley \& Sons], New York, 1978.

[16] M. Kontsevich, Homological algebra of mirror symmetry, in Proceedings of the International Congress of Mathematicians, Vol. 1, 2 (Zrich, 1994), 120-139, Birkhuser, Basel, 1995.

[17] M. Kontsevich, Deformation quantization of Poisson manifolds, I, preprint, q-alg/9709040.

[18] J.-L. Koszul, Crochet de Schouten-Nijenhuis et cohomologie, The mathematical heritage of Élie Cartan (Lyon, 1984), Astrisque 1985, Numero Hors Serie, 257-271.

[19] J. Li, G. Tian, Comparison of the algebraic and the symplectic Gromov-Witten invariants, preprint, alg-geom/9712035.

[20] B. Lian, K. Liu, S.T. Yau, Mirror principle I, Asian J. Math 1 (1997), no. 4, 729-763, alg-geom/9712011.

[21] G. Liu, Associativity of quantum multiplication, Comm. Math. Phys. 191 (1998), no. 2, 265-282.

[22] D. McDuff, D. Salamon, J-holomorphic curves and quantum cohomology, University Lecture Series, 6, American Mathematical Society, Providence, RI, 1994. 
[23] Yu. Manin, Frobenius manifolds, quantum cohomology, and moduli spaces, AMS Colloquium Publications, 47, AMS, Providence, RI, 1999.

[24] Y. Manin, Three constructions of Frobenius manifolds: a comparative study, preprint, math.QA/9801006.

[25] D.R. Morrison, Mirror symmetry and rational curves on quintic threefolds: a guide for mathematicians, J. Amer. Math. Soc. 6 (1993), no. 1, 223-247.

[26] J. Morrow, K. Kodaira Complex manifolds, Holt, Rinehart and Winston, Inc., New York-Montreal, Que.-London, 1971.

[27] Z. Ran, Thickening Calabi-Yau moduli spaces, in Mirror symmetry, II, 393-400, AMS/IP Stud. Adv. Math., 1, Amer. Math. Soc., Providence, RI, 1997.

[28] Y. Ruan, G. Tian, A mathematical theory of quantum cohomology, J. Differential Geom. 42 (1995), no. 2, 259-367.

[29] A. Schwarz, Geometry of Batalin-Vilkovisky quantization, Comm. Math. Phys. 155 (1993), no. 2, 249-260.

[30] G. Tian, Smoothness of the universal deformation space of compact Calabi-Yau manifolds and its Petersson-Weil metric, in Mathematical aspects of string theory (San Diego, Calif., 1986), 629-646, Adv. Ser. Math. Phys., 1, World Sci. Publishing, Singapore, 1987.

[31] G. Tian, Quantum cohomology and its associativity, in Current developments in mathematics, 1995 (Cambridge, MA), 361-401, Internat. Press, Cambridge, MA, 1994.

[32] A.N. Todorov, The Weil-Petersson geometry of the moduli space of $\mathrm{SU}(n \geq 3)$ (Calabi-Yau) manifolds. I., Comm. Math. Phys. 126 (1989), no. 2, 325-346.

[33] C. Vafa, Topological mirrors and quantum rings, in Essays on mirror manifolds, S.T. Yau ed., 96-119, Internat. Press, Hong Kong, 1992.

[34] I. Vaisman, Lectures on the geometry of Poisson manifolds. Progress in Mathematics, 118, Birkhuser Verlag, Basel, 1994.

[35] E. Witten, Two-dimensional gravity and intersection theory on moduli space, in Surveys in differential geometry (Cambridge, MA, 1990), 243-310, Lehigh Univ., Bethlehem, PA, 1991.

[36] E. Witten, Mirror manifolds and topological field theory, in Essays on mirror manifolds, S. T. Yau ed., International Press Co., Hong Kong, 1992.

[37] S.T. Yau ed., Essays on mirror manifolds, International Press, Hong Kong, 1992. 
[38] E. Witten, A note on antibracket formalism, Modern Phys. Lett. A 5 (1990), no. $7,487-494$.

RECEIVED June 18, 1998.

TeXas A \& M University

College Station, TX 77843

E-mail addresses: cao@math.tamu.edu, zhou@math.tamu.edu 